\title{
Successful Outcome in Perinatal Intravaginal Torsion of Testis in Neonate: Long-Term Outcome
}

\author{
Kashif Chauhan ${ }^{a}$,, Gemma Bown ${ }^{a}$, Brian W. Davies ${ }^{a}$, Shailinder J. Singh ${ }^{a}$
}

\begin{abstract}
Perinatal testicular torsion can be intravaginal or extravaginal. Extravaginal torsion can be managed in an elective manner. Intravaginal torsion needs an urgent operation to maximize the viability of the testis. The history is vital to distinguish between the two diagnoses. We report a case in which a perinatal intravaginal torted testicle was successfully salvaged due to a timely exploration. This was a retrospective review of a case and literature review of perinatal testicular torsion. A term baby was transferred to a tertiary pediatric surgical unit in the United Kingdom for surgical management of exomphalos minor. The child was noted to have normal testes. On the seventh day of life, he was noted to have a firm swelling in his right scrotum with purple discoloration. He was promptly reviewed by the surgical team. A perinatal torsion of intravaginal type was suspected and he was booked for emergency exploration. The surgical findings were 1) significant edema of the right scrotal wall, 2) a thickened tunica vaginalis and small volume of hemolyzed fluid, and 3) a bluish and congested torted testicle in intravaginal plane. Testis was de-rotated and color returned within 5 minutes. A three-point testicular fixation was performed bilaterally. He was reviewed in clinic for the following 2 years and found to have equal growth of the testicles, both of which were appropriately positioned within the scrotum. This case highlights the importance of being aware that perinatal torsion can be extravaginal or intravaginal. The patient history is important to distinguish between the two diagnoses as proven by the above case. A positive outcome can be achieved with judicious assessment and emergent management of perinatal intravaginal torsions. Clinicians should maintain a high level of suspicion of intravaginal torsion in all cases of perinatal testicular torsion.
\end{abstract}

Keywords: Testicular torsion; Neonatal torsion; Intravaginal; Extravaginal

\section{Introduction}

Perinatal testicular torsion is often defined as occurring up to

Manuscript accepted for publication April 27, 2016

aPaediatric Surgery Department, Nottingham University Hospital, Nottingham, UK

${ }^{b}$ Corresponding Author: Kashif Chauhan, Paediatric Surgery Department, Nottingham University Hospital, Nottingham, UK.

Email: mkashifdr@yahoo.com

doi: http://dx.doi.org/10.14740/ijcp250e
30 days following birth, with $70-75 \%$ occurring intrauterine and $25-30 \%$ occurring in the postnatal period. Perinatal testicular torsion can be intravaginal or extravaginal, depending on whether there is involvement of the tunica vaginalis. Whilst extravaginal torsion can be managed in an elective manner, intravaginal torsion needs an urgent operation to maximize the viability of the testis. The history is vital to distinguish between the two diagnoses, yet management remains variable both in clinical practice and in literature. The reporting of successful testicular salvage remains low. We report a case in which a perinatal intravaginal torted testicle was successfully salvaged due to a timely exploration.

\section{Case Report}

A term baby was transferred to a tertiary pediatric surgical unit in the United Kingdom for surgical management of exomphalos minor. On arrival, the child was noted to have normal testes. On the seventh day of life, he was found to have a firm swelling in his right scrotum with purple discoloration. He was promptly reviewed by the surgical team. A perinatal torsion of intravaginal type was suspected and he was booked for emergency exploration and the operation was performed the same night. Less than $8 \mathrm{~h}$ passed between clinical suspicion of abnormality and surgical repair. Following features were found intra-operatively: significant edema of the right scrotal wall, a thickened tunica vaginalis and small volume of hemolyzed fluid, a bluish and congested torted testicle in intravaginal plane. The testis was de-rotated and color returned within $5 \mathrm{~min}$. The contralateral testis was also assessed and no abnormality was detected. A three-point testicular fixation was performed bilaterally. He was reviewed in clinic for the following 2 years and found to have equal growth of the testicles, both of which were appropriately positioned within the scrotum. We reviewed him again after 8 years and both the testis are lying comfortably in the scrotum (Fig. 1). They are both equally developed with normal lie, shape and appropriate size according to age (Fig. 1) .

\section{Discussion}

The consequence of late management of testicular torsion can be orchidectomy and reduced fertility. Therefore, it is pertinent that prompt assessment of this risk is performed. There are many reports in the literature of missed opportunities to 

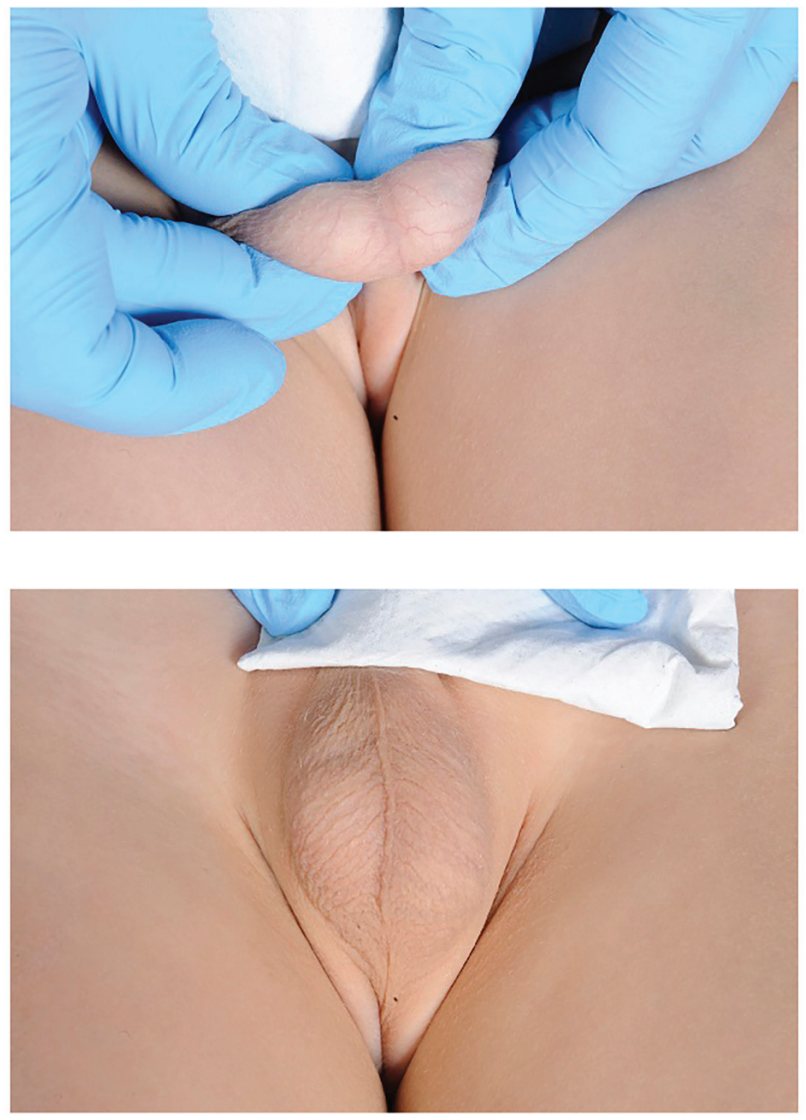

Figure 1. Picture of testis after 8 years of operation.

salvage the testicle following neonatal torsion, but we unusually show that with timely assessment, there can be no sequelae following this event.

The current literature shows clinicians are less likely to perform emergency surgery on perinatal torsions compared to older children [1]. This could be due to differences in presentation. The majority of perinatal torsions are extravaginal, with twisting of both the spermatic cord and tunica vaginalis, whereas the majority of torsions in older children are intravaginal. Furthermore, the majority of perinatal torsions are intrauterine, with the clinical picture varying depending on time of torsion.

The importance of timely exploration of acute scrotal findings is two-fold: firstly increased likelihood of maintaining testicular volume and function and secondly high incidence of asynchronous bilateral perinatal torsion. When torsion occurs earlier in pregnancy, the child may present with an absent or nubbin testicle only or with a firm, painless mass high up in the scrotum with no acute inflammation [2]. Histological review also shows reduced size compared to contralateral testis and increased proportion of fibrotic tissue formed. If torsion occurs in the days before birth, the presentation is likely of a firm, painless mass, closer in size to the contralateral testis. Postnatal torsion presentation is similar to that in puberty of a painful, swollen testicle with a discoloured hemiscrotum.

Prenatal torsions are unlikely to be salvageable due to prolonged period of intrauterine ischemia. Therefore, clinically these are often managed conservatively until approximately 1 month of age, when the anesthetic risks are reduced. It is important to note on assessment that the common features are of non-painful, non-inflammed testis which is present at birth. Conversely, postnatal torsions can successfully be salvaged and should be managed as for older children, especially if a normal appearance is noted following birth. This highlights the importance of clear documentation of testicular appearance following birth as has occurred in this case. With any suspicion of acute perinatal torsion emergency exploration of the testis is recommended by the European Association of Urology [1].

Bilateral perinatal torsion accounts for up to $33 \%$ of all perinatal torsions [3]. Furthermore, up to $33 \%$ of these are asynchronous (onset of symptoms separated over time), usually with normal appearance of the contralateral testis on initial presentation. However, the timing of secondary torsion is unpredictable and has been reported as quickly as $48 \mathrm{~h}$ following initial torsion [4]. Although the risk is small, this raises this question of whether early surgery is recommended in all perinatal torsions in order to reduce the risk of contralateral torsion and anorchia.

\section{Conclusion}

This case highlights the importance of being aware that perinatal torsion can be extravaginal or intravaginal. The patient history is important to distinguish between the two diagnoses as proven by the above case. A positive outcome can be achieved with judicious assessment and documentation and emergent management of intravaginal torsions. Clinicians should maintain a high level of suspicion of intravaginal torsion in all cases of perinatal testicular torsion.

\section{Funding}

There was no funding for this case report.

\section{Conflicts of Interest}

All the authors declare that they have no conflicts of interest.

\section{References}

1. Riaz-Ul-Haq M, Mahdi DE, Elhassan EU. Neonatal testicular torsion; a review article. Iran J Pediatr. 2012;22(3):281-289.

2. Ashraf I, Ibrahim A, Bazeed M, Hader H, Raheem IMA. Perinatal testicular torsion: literature review and local experience. Annals of Pediatric Surgery. 2012;8:86-89.

3. Kaye JD, Levitt SB, Friedman SC, Franco I, Gitlin J, Palmer LS. Neonatal torsion: a 14-year experience and proposed algorithm for management. J Urol. 
2008;179(6):2377-2383.

4. Baglaj M, Carachi R. Neonatal bilateral testicu- lar torsion: a plea for emergency exploration. J Urol. 2007;177(6):2296-2299. 